\title{
Effects of Dietary Approaches to Stop Hypertension (DASH) diet on cardiovascular risk factors: a systematic review and meta-analysis
}

\author{
M. Siervo, J. Lara, S. Chowdhury, C Oggioni, D. A. Ashor and J. C. Mathers \\ Human Nutrition Research Centre, Institute for Ageing and Health, Newcastle University, Biomedical Research \\ Building, Campus for Ageing and Vitality, Newcastle Upon Tyne, NE4 5PL, UK
}

Background: The Dietary Approach to Stop Hypertension (DASH) is recommended to lower blood pressure (BP) but effects on cardiovascular risk factors are unclear. We conducted a systematic review and meta-analysis of randomised controlled trials (RCTs) to test the effects of the DASH diet on cardiovascular risk factors.

Methods and Results: Medline, EMBASE, and Scopus databases were searched from inception to December 2013. Inclusion criteria were: 1)DASH diet; 2)RCTs; 3)risk factors including systolic and diastolic BP, glucose, high-density lipoproteins (HDL), low-density lipoproteins (LDL), triglycerides and total cholesterol; and 4)control group. Random-effects models were used to determine the pooled effect sizes. Meta-regression analysis examined the associations between the effect size and baseline values of the cardiovascular risk factors, body mass index (BMI), age, quality of trials and study duration.

Results: Twenty articles reporting data for 1,917 participants were included in the meta-analysis. Intervention duration ranged from 2 to 24 weeks. The DASH diet produced significant decreases in systolic $(-4.5 \mathrm{mmHg}, 95 \% \mathrm{CI}=-5 \cdot 7-3 \cdot 2, p<0 \cdot 001$, Figure 1$)$ and diastolic BP $(-2.5 \mathrm{mmHg}, 95 \% \mathrm{CI}=-3.3-1.7, p<0.001)$ and concentrations of total cholesterol $(-7.6 \mathrm{mg} / \mathrm{dL}, 95 \% \mathrm{CI}=-12.5$ $-2.8, p=0.002)$, LDL $(-4.9 \mathrm{mg} / \mathrm{dL}, 95 \% \mathrm{CI}=-8.9-0.8, p=0.01)$ and glucose $(-3.0 \mathrm{mg} / \mathrm{dL}, 95 \% \mathrm{CI}=-5.5-0.6, p=0.01)$. Changes in both systolic and diastolic BP were greater in participants with higher baseline BP or BMI. These changes in cardiovascular risk factors predicted $\sim 12 \%$ reduction in 10-year risk Framingham risk score for cardiovascular diseases (Figure 1).
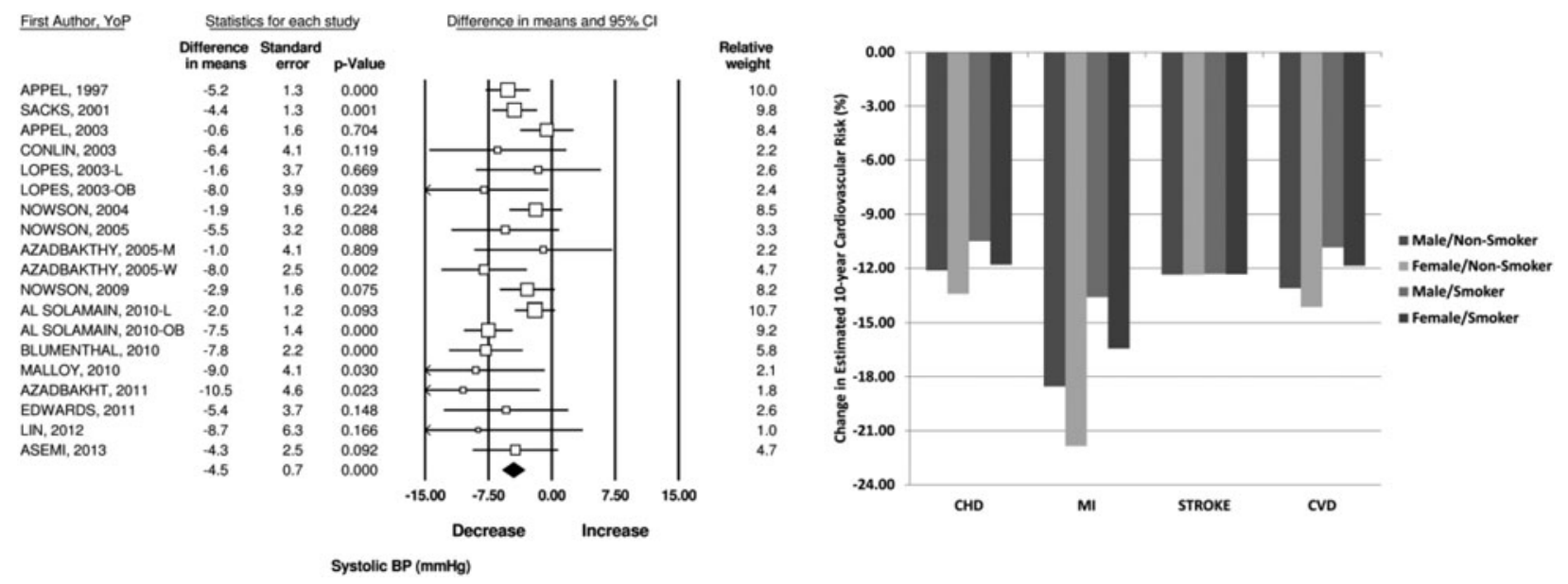

Fig. 1. Forest-plots of randomized clinical trials investigating the effects of DASH dietary interventions on systolic pressure (BP, left panel) and predicted percent change in 10-year probability of developing a cardiovascular event as a consequence of changes in systolic blood pressure, total cholesterol and HDL from the meta-analysis data (right panel).

Conclusions: The DASH diet improved cardiovascular risk factors and appeared to have greater benefits in subjects at higher cardiometabolic risk. The DASH diet is an effective nutritional strategy to lower cardiovascular risk. 